$\begin{gathered}\text { Revista do Departamento de Geografia } \\ \text { Universidade de São Paulo } \\ \text { www.revistas.usp.br/rdg } \\ \text { Vovista do Departamento de Geografia USP } \\ \text { ISSN 2236-2878 }\end{gathered}$
Volume Especial (2016)

\title{
ONDAS DE FRIO E ENFERMIDADES RESPIRATÓRIAS: ANÁLISE NA PERSPECTIVA DA VULNERABILIDADE CLIMÁTICA
}

\author{
COLD WAVES AND RESPIRATORY DISEASE: ANALYSIS FROM CLIMATE \\ VULNERABILITY PERSPECTIVE
}

\author{
Karime Pechutti Fante \\ Universidade Estadual Paulista Julio de Mesquita Filho \\ karimefante@hotmail.com \\ Núbia Beray Armond \\ Universidade Estadual Paulista Julio de Mesquita Filho \\ nubia.beray@gmail.com
}

Resumo: As repercussões das dinâmicas dos elementos atmosféricos no território são deflagradas, fundamentalmente, pelas formas através das quais o espaço geográfico é produzido pelos grupos sociais. Nesse contexto, o artigo procurou abordar a relação entre o conceito de eventos extremos e vulnerabilidade a enfermidades respiratórias utilizando-se de estudo exploratório sobre ondas de frio em Piracicaba - São Paulo. Para a sua identificação, foram utilizados cinco índices climáticos diferentes aplicados aos dados de temperaturas mínimas, obtidos pelo INMET. As ondas de frio detectadas em cada um dos índices foram correlacionadas (comparativamente) com os dados de internação por doenças respiratórias obtidos no DataSUS, entre os anos de 1998 e 2014. Os resultados indicam a ocorrência de maior número de ondas de frio nos meses de inverno, ocorridas principalmente no período da normal climatológica (1961-1990) quando comparada ao período recente (1991-2014). Entre as técnicas comparadas, observou-se que a quantidade de ondas identificadas é díspar e varia de 72 (CSDI-OMM) a 329 ondas (IPCC) na mesma série histórica. Na análise da vulnerabilidade, foi identificado que a faixa etária mais afetada por enfermidades respiratórias é de 0 a 9 anos e acima de 80 anos, mais da metade do total de internações entre 1998 a 2014 (21.115 internações). De modo geral, os meses representativos do inverno, que havia apresentado maior ocorrência de ondas de frio, também registraram maior número de internações, principalmente o mês de julho, com uma média superior a 200 internações. Por fim, os resultados indicam que não é apenas a temperatura (onda de frio) a principal responsável pela deflagração de enfermidades respiratórias. Concluiu-se a necessidade de se abordar os conceitos de eventos extremos e vulnerabilidade para uma análise socioespacial da produção das doenças respiratórias, onde os elementos climáticos consistem em apenas um fator para a deflagração dessas doenças.

Palavras-chave: Ondas de frio; Enfermidades respiratórias; Vulnerabilidade; Índices climáticos.
Abstract: Different ways of geographic space production by social groups deflagrates different repercussions of climate dynamics by atmospheric elements. In this context, this article aims to approach the relationship between extreme events and vulnerability to respiratory diseases from an exploratory study of cold waves in Piracicaba - São Paulo - Brazil. Five different climatic indices were applied to minimum temperature data from an INMET station. 1998-2014-respiratory diseases hospitalization data from DataSUS were correlated comparatively to cold waves given in each technique. Results indicate increases in cold waves occurrence in winter months, especially in the climatological normal period (1961-1990) in comparison to the recent period (1991-2014). Between the different techniques, the cold waves number is uneven and varies from 72 cold waves (CSDI-OMM index) to 329 (IPCC index) in the same period. Vulnerability analysis demonstrated $0-9$ and $80+$ years old people represents the more affected public for respiratory diseases, representing more than $50 \%$ of all the hospitalizations $(21,115)$. In general, the winter months in which presented more cold waves totals also presented more hospitalizations, especially in July, with more than 200 hospitalizations. The results show that temperature (cold wave) is not the principal responsible to respiratory diseases deflagration. There is the need to approach extreme event and vulnerability concepts to a sociospatial analysis of respiratory diseases production, in which the climatic elements are only one factor in the diseases deflagration.

Keywords: Cold waves; Respiratory diseases; vulnerability; Climatic indices. 


\section{INTRODUÇÃO}

O homem e a maioria dos seres vivos são extremamente sensíveis a mudanças e alterações no ambiente em que vivem. De modo intrínseco e indissociável, a interação entre sociedade e natureza revela-se como um híbrido e assume um papel importante nas condições atuais de vida e de bem-estar.

Silva, Ribeiro e Santana (2014) ressaltam que o organismo humano está inserido na atmosfera, numa complexidade de interações em estado permanente de confrontação para a manutenção das suas funções vitais. Essa relação se dá, principalmente, a partir do equilíbrio entre a produção e a perda de calor em resposta às mudanças dos estados químico e físico da atmosfera.

A interação atmosfera-superfície torna-se um elemento chave para o entendimento e correlação da influência do meio ambiente com o organismo humano. Condições específicas de temperatura, umidade, pressão, ventos, precipitação e a própria poluição atmosférica são elementos que interferem direta e indiretamente nas condições de saúde e funcionamento do organismo humano.

Ainda que não possam ser considerados como fatores únicos das condições de saúde humana, os elementos atmosféricos, quando associados às características físicas, biológicas, psicológicas e culturais, particulares de cada indivíduo, podem agravar e auxiliar no desencadeamento de doenças e enfermidades cardiovasculares (BRAGA et al. 2007; NOGUEIRA, 2009; FREITAS et al. 2013; MURARA, 2009 e 2012); respiratórias (SOUZA e SANT'ANNA NETO, 2008; SOUZA, 2008; ARAÚJO, 2014), e infecciosas de veiculação hídrica (ALEIXO, 2012).

Em específico as doenças relacionadas aos problemas respiratórios, foco desta pesquisa, Tortora (2000) apud Souza (2008, p. 59) relembra que as enfermidades respiratórias mais comuns são câncer de pulmão, a asma, a bronquite, o enfisema, a pneumonia, a tuberculose (TB), a síndrome da angústia respiratória (SAR) do recém-nascido, a insuficiência respiratória, a síndrome da morte súbita do infante (SMSI), o resfriado comum e a influenza, a embolia pulmonar e o edema pulmonar.

Miranda et al. (1995) apud Sette e Ribeiro (2011, p.44) ressaltam que esses agravos e doenças respiratórias agudas e crônicas ocorrem principalmente durante os meses de inverno em São Paulo. Segundo as autoras, os atributos climáticos que mais geram essas condições são a queda da temperatura e da umidade do ar no inverno, a maior amplitude térmica diária, pouca insolação, oscilações bruscas de temperatura, quando da aproximação e passagem de frentes frias e redução da dispersão dos poluentes (SETTE e RIBEIRO, 2011, p.45). No entanto, apesar das temperaturas e baixa umidade do ar serem fundamentais nestas ocorrências, deve-se considerar que há uma interação de fatores que permitem tal conjuntura (Figura 1).

De acordo com Miranda (1995) apud Sette e Ribeiro (2011, p. 44) fatores como clima, tempo e formas do espaço, o uso e ocupação do solo, características do organismo dos indivíduos (homem, mulher, adulto, criança, idoso) e as condições de vida são alguns dos elementos que interagem e contribuem para as incidências de enfermidades de cunho respiratório. Assim, é necessário compreender como os elementos atmosféricos impactam os diferentes grupos a fim de que seja possível se precaver sobre tais incidências gerando, por exemplo, medidas adaptativas que garantam a todas as mesmas condições de qualidade de vida em relação, principalmente, aos eventos climáticos extremos.

Em específico às doenças respiratórias, a influência dos elementos atmosféricos na saúde não ocorre instantaneamente. Suas especificidades compreendem a exposição de indivíduos em situações alarmantes como, por exemplo, dias/noites com temperaturas abaixo/acima do habitual por uma sequência determinada de dias. Ao partir deste pressuposto, definir a partir de qual o limiar os elementos atmosféricos e, especialmente, as temperaturas passam a ser um risco à população não é tarefa fácil, pois há diferentes técnicas, índices e conceituações adotados na climatologia a fim de definir o que é confortável ou não à saúde pública e aos grupos sociais, em função das condições culturais e ambientais.

Ainda hoje não há um consenso sobre estas definições, uma vez que a utilização de diferentes índices e técnicas pode resultar em grandes diferenças de interpretação sobre os eventos climáticos extremos e a influência na saúde humana. 


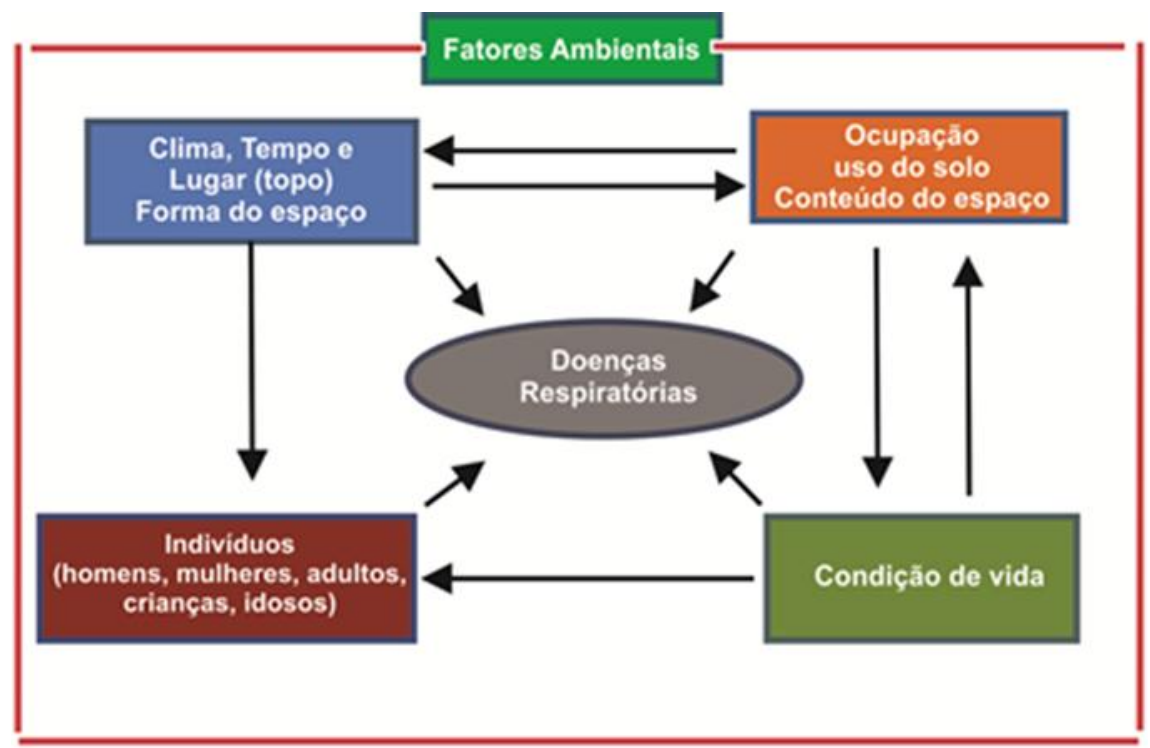

Figura 1: Interações entre os fatores ambientais e as doenças respiratórias crônicas. Fonte: Adaptado de Miranda et al. 1995, apud Sette e Ribeiro (2011, p. 44).

Acredita-se que considerar que a sazonalidade climática possui uma dinâmica própria (tanto nos seus padrões quanto nas suas excepcionalidades), e que os sujeitos se relacionam com estas dinâmicas de forma diferenciada no espaço (sendo mais ou menos vulneráveis), complexifica a relação entre os elementos do clima e os seus níveis de influência na saúde humana (SANT'ANNA NETO, 2001, 2008, 2011; SOUZA, 2008; ALEIXO, 2012).

Dessa forma, o artigo explora e relaciona os conceitos de evento extremo e vulnerabilidade através do estudo de caso das ondas de frio em Piracicaba (SP). Em detrimento às particularidades das diferentes metodologias analíticas sobre ondas de frio, objetivou-se analisar e comparar os índices climáticos que mais se adequam à verificabilidade da vulnerabilidade da população a episódios extremos. Neste caso, buscou-se relacionar os eventos de ondas de frio aos dados de internação mensal por enfermidades respiratórias entre os anos de 1998 a 2014.

\section{EVENTOS EXTREMOS E VULNERABILIDADE}

\section{Eventos Extremos}

Existe uma vasta literatura, sobretudo nas ciências que abordam o fenômeno climático, que se relacionam com os eventos extremos. Não é o objetivo do trabalho a realização de uma revisão bibliográfica exaustiva em busca de definições, mas sim partir da relação de algumas dessas definições para uma abordagem geográfica das ondas de frio.

Os eventos extremos, de forma geral, são considerados como anomalias ou desvios que flutuam em torno de um padrão médio. Constituem-se em fenômenos que se manifestam de forma diferente do seu padrão habitual, sobretudo estatisticamente, ou mesmo acima dos desvios padrões positivos e negativos utilizados para mensurar os elementos climáticos (ARMOND, 2014). Pode-se aplicar este pressuposto aos estudos de temperatura, nos quais se utiliza de medidas de tendência central, de frequência e de intensidade para gerar índices de identificação de grandes ondas de calor ou de frio.

No âmbito da climatologia, Monteiro e Carvalho (2013) esclarecem que há uma diferença fundamental na interpretação e utilização de índices climáticos. Alguns deles são utilizados na identificação da relação direta entre os elementos climáticos e a saúde e bem-estar dos seres humanos. A ênfase é dada ao conforto/desconforto térmico e suas implicações com o organismo humano. São conhecidos como os índices bioclimáticos. Podem-se citar, neste caso, os índices de desconforto de Thom e o Heat índex, a partir do qual são identificados níveis de perigo ao bem-estar e à saúde. 
Numa segunda vertente analítica, e diferente da anterior, os chamados índices climáticos pautam-se em valores de referência (quantitativos) para definir os critérios de excepcionalidade. A questão da influência na saúde ocorre indiretamente neste caso, pois se constata apenas o aumento/diminuição dos extremos térmicos que podem deflagrar situações negativas em relação à saúde pública. No entanto, esses índices pautam-se apenas na mensuração e quantificação dos eventos climáticos de temperaturas extremas.

Segundo o Instituto Português do Mar e Atmosfera, as técnicas utilizadas para a definição do que pode ser considerado extremo está mais relacionada com o estudo e análise da variabilidade climática (em termos de tendências) do que propriamente com os impactos na saúde pública de temperaturas extremas que possam observar-se num período mais curto.

Seja pelo viés direto dos índices bioclimáticos ou indiretos dos índices climáticos, o fato é que ambas as vertentes analíticas são importantes ferramentas no reconhecimento dos fenômenos atmosféricos extremos e se admite a necessidade de prevenção e adaptação das cidades brasileiras aos episódios atmosféricos excepcionais.

A Organização Meteorológica Mundial (OMM) e o Painel Intergovernamental de Mudanças Climáticas (IPCC), por exemplo, são partidários de análises a partir de índices climáticos. Para a OMM, conforme Monteiro e Carvalho (2013) define-se uma onda de calor quando há permanência de pelo menos seis dias consecutivos, com temperaturas máximas diárias superiores em $5^{\circ} \mathrm{C}$ ao valor médio diário do período de referência (média de um período, por exemplo).

Para o IPCC (2001), o critério adotado para definir o índice de duração de uma onda de calor é a ocorrência de, pelo menos, cinco dias consecutivos com temperaturas máximas diárias superiores em $5^{\circ} \mathrm{C}$ relativamente ao período de referência.

No Brasil, mais especificamente no Rio Grande do Sul, Silveira (2013) utilizou-se da técnica dos Quantis e também definiu limiares para a identificação de ondas de calor. Em sua abordagem, considerou quatro enfoques principais: evento extremo (Q90 e duração mínima de três dias), superevento (Q10 e no mínimo três dias de permanência), evento alerta (Q85 e episódios com duração mínima de três dias) e dia de alerta (Q85 e episódio com duração mínima de um dia), em cidades do Rio Grande do Sul.

Atrelado aos limiares quantitativos para a definição das ondas de frio, Monteiro (1991) realiza a aproximação do tratamento do clima na Geografia e na Meteorologia. A Geografia se guiaria em função das necessidades humanas cotidianas de extrair a vida do meio através da forma adequada de organização espacial.

Segundo Monteiro (1991)

\begin{abstract}
À medida que se percebem as correlações complexas e uma concepção mais comportamental e complexa do clima é que nos damos conta de como os estados de tempo oscilam, admitem desvios e produzem 'acidentes' que não podem ser considerados excepcionais. Mesmo os mais graves, por vezes catastróficos, se estatisticamente poderiam ser tomados como 'ruído', seu impacto local ou regional e a reação em cadeia que se lhes segue, fazem com que eles não possam ser descartados. E, sobretudo por suas repercussões socioeconômicas, tanto por injúrias imediatas como pelo efeito de 'desregularização' da produção agrícola, do sistema hidro-energético, da rede de transportes, etc (p. 19)
\end{abstract}

Para o autor, as repercussões de episódios que caracterizam os desvios de precipitação ou de temperatura, por exemplo, parecem ser o que une a relação sociedade e natureza na Climatologia Geográfica. Deste modo, o conhecimento e compreensão do evento extremo são essenciais para prevenção das situações de vulnerabilidade. Somente a partir da constatação e análise das ocorrências é que é possível gerir um sistema de alerta e medidas adaptativas que garantam melhores condições de conforto térmico e qualidade de vida aos diferentes grupos sociais e, especialmente, à camada mais vulnerável, que mais sofrem com os efeitos negativos das baixas temperaturas na saúde. 


\section{Vulnerabilidade}

O conceito de vulnerabilidade tem sido amplamente discutido na ciência geográfica principalmente a partir da Geografia Física e dos estudos de interface com o meio ambiente. Na literatura anglo-saxônica, o surgimento e popularização do conceito de hazards nos anos 1980 abriu uma série de possibilidades de estudos integradores entre as dinâmicas da natureza em relação com aquelas da sociedade (MONTEIRO, 1991). Uma constelação de conceitos, tributária dos hazards (azares) emergiu desde então. Dentre os principais, destaca-se a potência da dimensão espacial do conceito de vulnerabilidade.

Na ciência geográfica a vulnerabilidade tem sido definida e abordada, sobretudo com relação à chamada dimensão ambiental (CUTTER, 1996, 2003; LUERS, 2005; ADGER, 2006). Este conceito tem auxiliado a demonstrar as diferentes formas pelas quais as relações entre grupos sociais e a natureza se realiza (DOW, 1992; KATES, 1985 apud CUTTER, 1996; VEYRET, 2007), inclusive em sua dimensão espacial (CUTTER, 1996, 2003; LUERS, 2005; ADGER, 2006; ARMOND, 2014).

Para Dow (1992), a vulnerabilidade representa a capacidade diferencial de grupos e indivíduos em lidar com os hazards, tendo como base suas posições nas dimensões físicas e sociais. No caso de Kates (1985) apud Cutter (1996), a vulnerabilidade apresenta-se como a capacidade de sofrer danos e reagir às adversidades. Veyret (2007) ainda destaca a dimensão social do conceito de vulnerabilidade, que, para a autora, é compreendido como uma construção histórica.

Estas três definições exemplificam que a vulnerabilidade tem sido abordada sem abrir mão da consideração das diferenças sociais, econômicas, culturais e ambientais entre os grupos sociais em relação aos desastres e/ou catástrofes. Na verdade, ambas vão além de considerar apenas o grau de exposição diferenciada desses grupos. Pode-se inferir, principalmente a partir da definição de Veyret (2007), que a produção das diferenças entre as classes sociais é a mesma que produz a vulnerabilidade. Como se ao reproduzirem a sua existência enquanto classe social reproduzem de forma inerente as condições de vulnerabilidade a determinados hazards.

Para Adger (2006), a vulnerabilidade é um fenômeno dinâmico que relaciona fluxos biofísicos e processos sociais que ocorrem em determinadas condições locais, ou seja, a vulnerabilidade seria um indicador das formas pelas quais se dá a relação sociedade e natureza em determinado recorte espacial, seja ele local, microrregional, macrorregional, nacional etc. Além disso, para o autor, locais e grupos sociais vulneráveis são, de maneira geral, excluídos do processo de tomada de decisão política, o que acaba por perpetuar a sua condição de vulnerabilidade. Nesse caso, a produção da vulnerabilidade e a exclusão de grupos sociais estariam intimamente relacionadas (SATTERTHWAITE et al., 2009).

É importante ressaltar que as noções de vulnerabilidade não abordam os grupos sociais como agentes passivos na produção do potencial de ocorrência de desastres e/ou catástrofes. Pelo contrário: as dinâmicas de determinados grupos sociais por vezes são responsáveis pela própria produção da vulnerabilidade, strictu sensu. Pode-se depreender que, ainda que estejam situados em um mesmo grupo biofísico de risco (idosos e crianças), existem grupos sociais com maiores condições de se defender de fenômenos como eventos extremos de chuva, ondas de frio ou de calor.

Segundo Luers (2005), o objetivo primeiro dos estudos de vulnerabilidade é identificar as pessoas ou lugares que são mais susceptíveis aos danos para produzir ações de redução dessa vulnerabilidade. Cutter (1996, 2003) acrescenta a essas definições a dimensão espacial da vulnerabilidade. Para a autora, a vulnerabilidade está, em geral, relacionada a um lugar, sendo, portanto, inerente a dimensão geográfica.

Ao realizar uma considerável revisão bibliográfica, Cutter (1996) identifica no trabalho de Livermann (1990) a diferenciação entre a vulnerabilidade localizada (áreas vulneráveis ou locais onde se situam pessoas vulneráveis) e a vulnerabilidade no que denomina de "espaço social", em que diferencia os grupos sociais em uma determinada área (CIDADE, 2013; CUTTER, 1996).

$\mathrm{Na}$ esteira de todas as definições apresentadas até então, Confalonieri (2003) afirma a vulnerabilidade como a exposição de um grupo social a um perigo natural e a sua capacidade de se recuperar de um desastre. Para o autor, os impactos deflagrados a partir da variabilidade dos elementos do clima necessitam do entendimento dos atributos socioespaciais da vulnerabilidade. Apenas a partir da consideração de problemas como a instalação de habitações em locais inadequados, da ausência de políticas de mobilidade 
urbana, da efetivação de políticas de tratamento de resíduos, entre outros, é que se pode produzir conhecimentos para a mitigação desses impactos de forma eficaz.

Mesmo no caso das morbidades respiratórias em sua relação com a dinâmica climática, o conceito de vulnerabilidade se adequa à análise, pois se apresenta tributário de e demanda uma abordagem multidimensional, que não abra mão da identificação de: (a) quem são os sujeitos vulneráveis; (b) onde estão os sujeitos vulneráveis; (c) quando/em que situações estes sujeitos são vulneráveis.

Ressalta-se que a geograficidade creditada ao conceito de vulnerabilidade se dá não apenas por conta da sua inerente dimensão espacial (CUTTER, 1996, 2003; CIDADE, 2013), mas principalmente pela sua utilização ampla na análise integrada entre sociedade e natureza. Respectivamente, trata-se da principal categoria geográfica e do eixo epistemológico da Geografia. Além disso, tanto o evento extremo como a vulnerabilidade são abordados, quase em sua totalidade, em relação com a sociedade. Em outras palavras, não há eventos extremos e nem vulnerabilidade se ambas não afetarem, de alguma maneira, as dinâmicas sociais (SMITH e PETLEY, 2007). Essas três características aproximam e tornam a vulnerabilidade um conceito eminentemente geográfico.

\section{ÁREA DE ESTUDO}

Situada ao centro do estado de São Paulo e com população estimada em 364.872 habitantes, a cidade de Piracicaba localiza-se a 547 m de altitude do nível do mar. Segundo Ferraz et al. (1998, p. 17), Piracicaba (...) está localizada em uma das regiões mais ricas e urbanizada do país, dotada de características econômicas diversas, as quais podem ser divididas em três grupos: agroindústria, área canavieira local e o entorno industrial metropolitano (Figura 2).

De acordo com Fante (2014) as médias neste local são de $28,4^{\circ} \mathrm{C}$ para as temperaturas máximas e $15,2^{\circ} \mathrm{C}$ para as temperaturas mínimas.

Durante o ano na maior parte dos dias (aproximadamente 150 dias) as temperaturas máximas ocorrem entre $27^{\circ}$ a $31^{\circ} \mathrm{C}$. Para as mínimas, habitualmente (cerca de $1 / 3$ do ano), as temperaturas variam entre $16,1^{\circ}$ a $20^{\circ} \mathrm{C}$.

Os maiores extremos quentes e frios ocorrem nos meses representativos de verão (dezembro a março, com ênfase para o ápice das máximas em fevereiro, média de $30,7^{\circ} \mathrm{C}$ ) e inverno (junho a agosto, sendo que as médias das temperaturas mínimas mais expressivas ocorreram durante o mês de julho, $11,1^{\circ} \mathrm{C}$ ).

\section{PROCEDIMENTOS METODOLÓGICOS}

Os dados utilizados na pesquisa foram obtidos junto ao banco climatológico da Escola Superior de Agricultura Luiz de Queiroz da Universidade de São Paulo (ESALQ/USP). Foram utilizados os dados diários de temperatura mínima dos anos de 1961 a 2014 para a comparação das técnicas de índices climáticos relativos.

Identificados os dias com valores superiores a estes limiares, buscou-se observar o total de eventos ao longo da série e também comparar os resultados entre diferentes períodos (normal climatológica e período recente). $\mathrm{O}$ objetivo foi constatar aumentos e/ou diminuições no número de ocorrências durante esses dois períodos e também comparar os resultados entre as técnicas.

A metodologia escolhida e aplicada nesta pesquisa foi a identificação de ondas de frio por meio de índices climáticos relativos. Optou-se pelas definições de cinco técnicas que priorizam intensidades e permanências de temperaturas extremas diferentes. Testaram-se as técnicas da Organização Meteorológica Mundial (OMM); Painel Intergovernamental de Mudanças Climáticas; índice australiano; a técnica elaborada por Silveira (2013) e a técnica intitulada Cold Speel Duration Index (CSDI-OMM), elaborada pela OMM. 


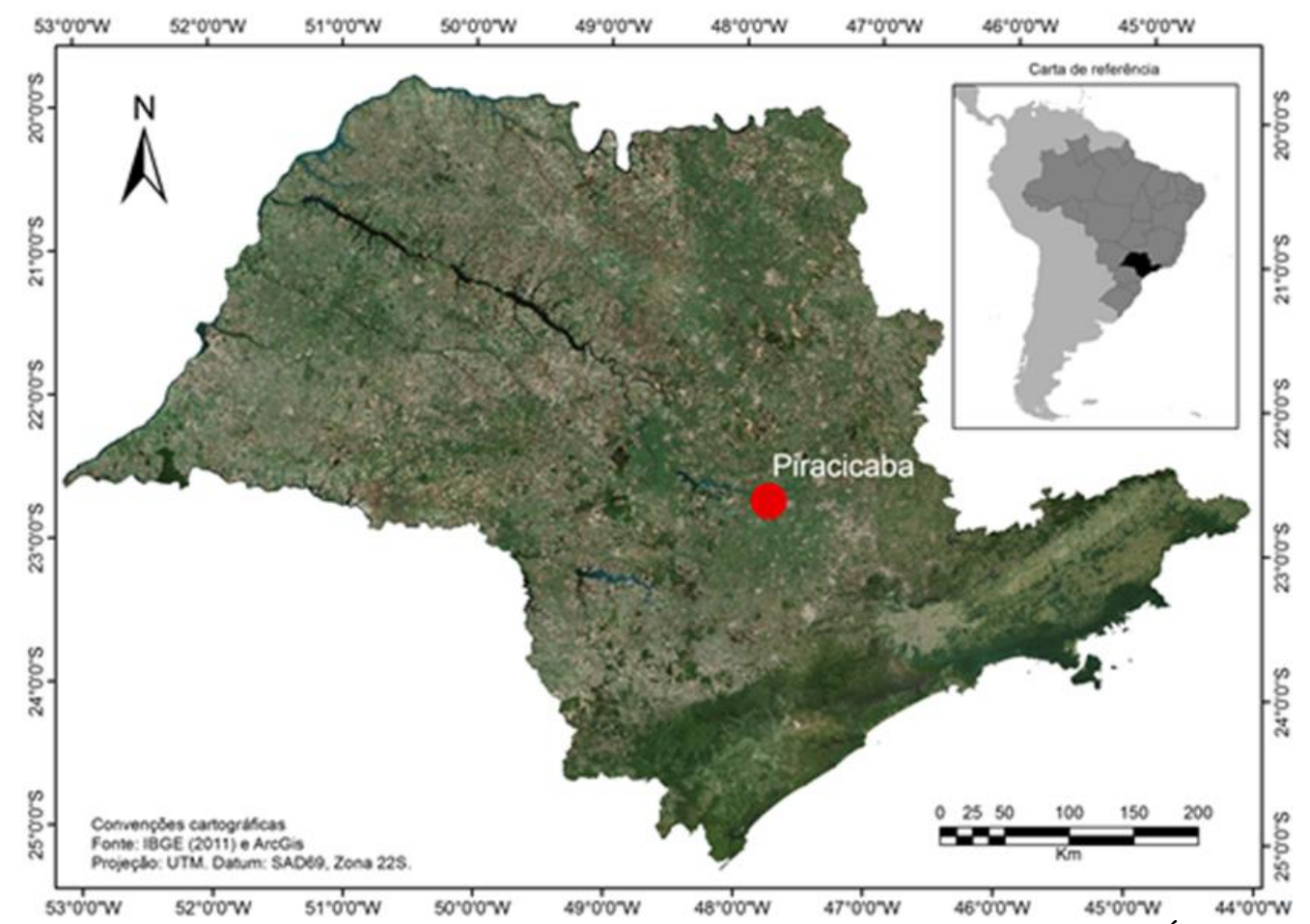

Figura 2: Localização do município de Piracicaba - SP. Organização: NASCIMENTO JÚNIOR, L.

Para cada técnica, assim como apresentado no Quadro 1, os valores considerados como extremos para a cidade de Piracicaba foram diferentes. Os valores de referência necessários para a aplicação dos índices da OMM e IPCC, foram calculados a partir da média das temperaturas mínimas da normal climatológica (1961-1990), que corresponde a $15,3^{\circ} \mathrm{C}$. Para o índice australiano, CSDI-OMM e Silveira (2013) o valor do percentil 10 foi $9,2^{\circ} \mathrm{C}$.

Os dados de internação por doenças e enfermidades do aparelho respiratório foram obtidos junto ao sistema de Classificação Estatística Internacional de Doenças e Problemas relacionados à saúde (CID-10) no banco de dados DataSUS (http://www.datasus.gov.br/cid10/V2008/cid10.htm). Para este conjunto de dados, as informações são disponibilizadas em totais mensais e por faixa etária desde o ano de 1998 a 2014.

Tais dados foram analisados mensalmente e por faixa etária buscando identificar os meses em que ocorre o maior número de internações e quais são os grupos mais vulneráveis. Também foram realizadas análises anuais a fim de comparar o número de ondas frio (eventos extremos) com o número de internações (vulnerabilidade).

Quadro 1: Índices climáticos relativos utilizados na pesquisa e os critérios de excepcionalidade.

\begin{tabular}{|c|c|}
\hline ÍNDICES CLIMÁTICOS RELATIVOS & CRITÉRIOS DE EXCEPCIONALIDADE \\
\hline Organização Meteorológica Mundial (OMM) & $\begin{array}{l}\text { Pelo menos seis dias consecutivos com temperatura } \\
\text { mínima menor ou igual a } 5^{\circ} \mathrm{C} \text { em relação em relação a } \\
\text { temperatura média diária do período de referência. }\end{array}$ \\
\hline $\begin{array}{l}\text { Painel Intergovernamental de Mudanças Climáticas } \\
\text { (IPCC) }\end{array}$ & $\begin{array}{l}\text { Pelo menos cinco dias consecutivos com temperatura } \\
\text { mínima menor ou igual a } 5^{\circ} \mathrm{C} \text { em relação a temperatura } \\
\text { média diária do período de referência. }\end{array}$ \\
\hline Índice australiano & $\begin{array}{l}\text { Pelo menos quatro noites com a temperatura mínima } \\
\text { inferior ao percentil } 10 \text { da série atual. }\end{array}$ \\
\hline Silveira (2013) & $\begin{array}{c}\text { Quatro categorias: evento extremo (Quantil } 90 \text { com } \\
\text { duração mínima de três dias), superevento (Quantil } 10 \\
\text { com, no mínimo, três dias de permanência), evento alerta } \\
\text { (Quantil } 85 \text { e episódios com duração mínima de três dias) } \\
\text { e dia alerta (Quantil } 85 \text { e episódio com duração de um } \\
\text { dia). }\end{array}$ \\
\hline Cold Speel Duration Index (CSDI-OMM) & $\begin{array}{c}\text { Pelo menos seis dias consecutivos com temperaturas } \\
\text { mínimas inferiores ao percentil } 10 .\end{array}$ \\
\hline
\end{tabular}

Fonte: Monteiro e Carvalho, 2013; Silveira, 2013. Organização: Fante e Armond (2016). 


\section{RESULTADOS}

Cada uma das técnicas foi aplicada para os dados de temperaturas mínimas para a obtenção dos meses de ocorrência e da frequência das ondas de frio. Dentre os demais, o índice do IPCC foi o que apresentou maior número de ondas na série histórica (1998-2014), com um total de quase 100 ondas no mês de agosto. Os índices da OMM e de Silveira (2013) se aproximaram de 90 ondas, respectivamente nos meses de maio e setembro, e no mês de julho. $O$ índice australiano apresentou 60 ondas no mês de julho, seguido de aproximadamente 40 ondas do índice CSDI-OMM, também no mês de julho (Figura 3).

De modo geral esse resultado se justifica devido as singularidades e prioridades enfatizadas por cada técnica. Os índices da OMM e do IPCC, por exemplo, valorizam a permanência de dias com temperaturas mais baixas (5, 6 dias), enquanto que, para o índice australiano, Silveira (2013) e CSDI-OMM o destaque é dado aos valores mais extremos da série (percentil 10).
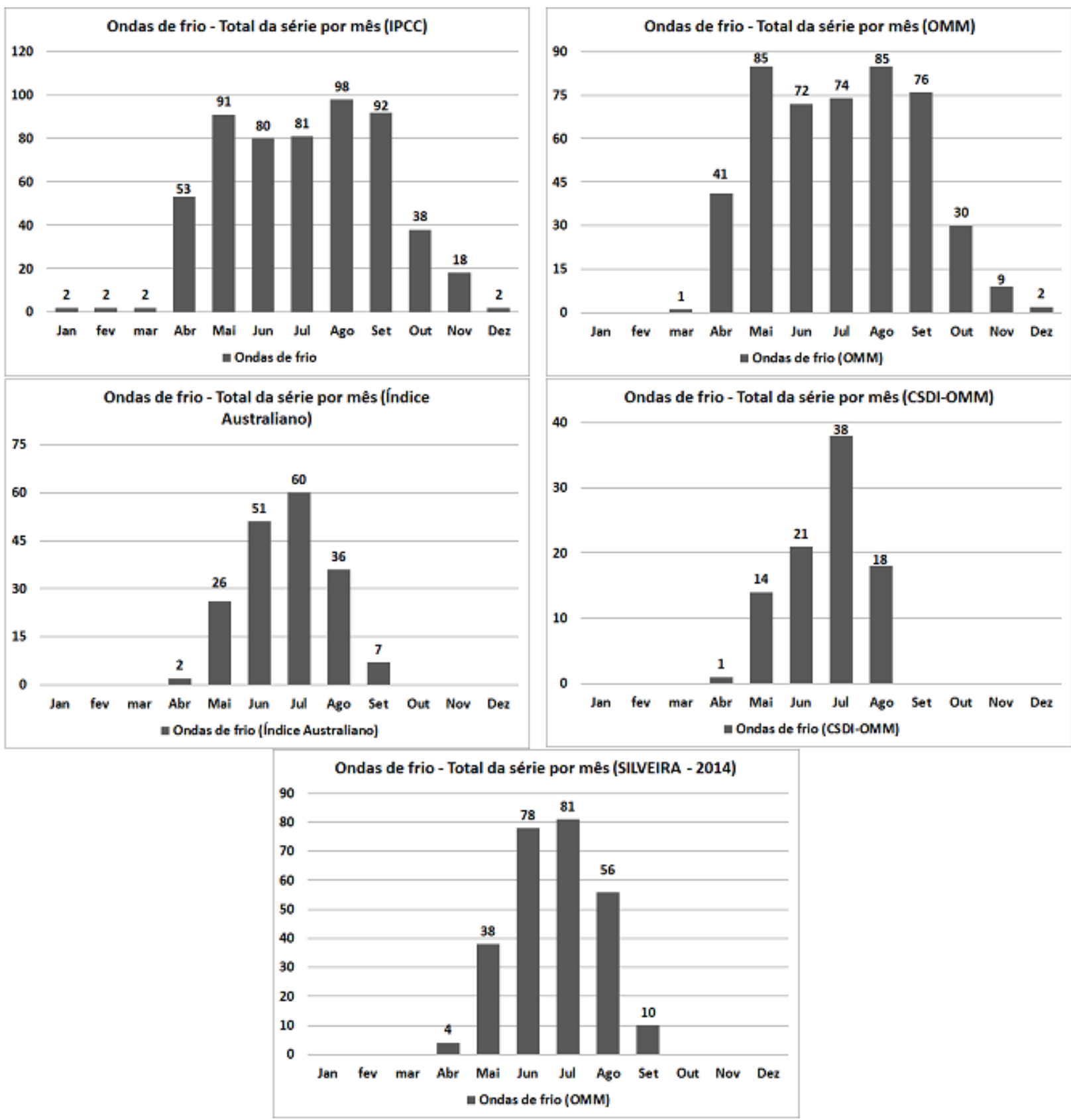

Figura 3: Totais de ondas de frio por mês na série histórica, segundo cada uma das técnicas empregadas. Fonte: Estação Meteorológica da ESALQ/USP. Organização: Fante e Armond (2016). 
O maior número de ondas de frio foi encontrado na estação menos chuvosa. A sua curva de ocorrência indica que elas começam no final do outono e terminam, em geral, no início da primavera. Há alguma variação temporal das ondas de frio entre os índices. A técnica empregada pelo IPCC identificou ondas de frio em todos os meses do ano, enquanto, através do índice da OMM, foram identificadas ondas em 10 meses (com exceção de janeiro e fevereiro).

Esse resultado está em consonância com a dinâmica climática sazonal para a faixa latitudinal em estudo. O início do outono marca o início das passagens frontais, que trazem na sua retaguarda o ar polar, que, ao diminuir as temperaturas e chegar a São Paulo com menos umidade, caracteriza o período de inverno (NIMER, 1989; SANT'ANNA NETO, 2005).

Em relação ao número de ondas de frio ocorridas durante o período da normal climatológica (1961 a 1990) e o recente (1991 - 2014) destaca-se que assim como encontrado na literatura (FANTE, 2014; DUFEK E AMBRIZZI, 2005; MINUZZI, CARAMORI E BORROZINO, 2011; COLLINS, CHAVES e MARQUES, 2009) houve um aumento nas médias das temperaturas mínimas e temperaturas extremas no município de Piracicaba, repercutindo, consequentemente, na diminuição do número de ondas de frio, nos últimos anos, fato este constatado em todos os índices utilizados (Figura 4).

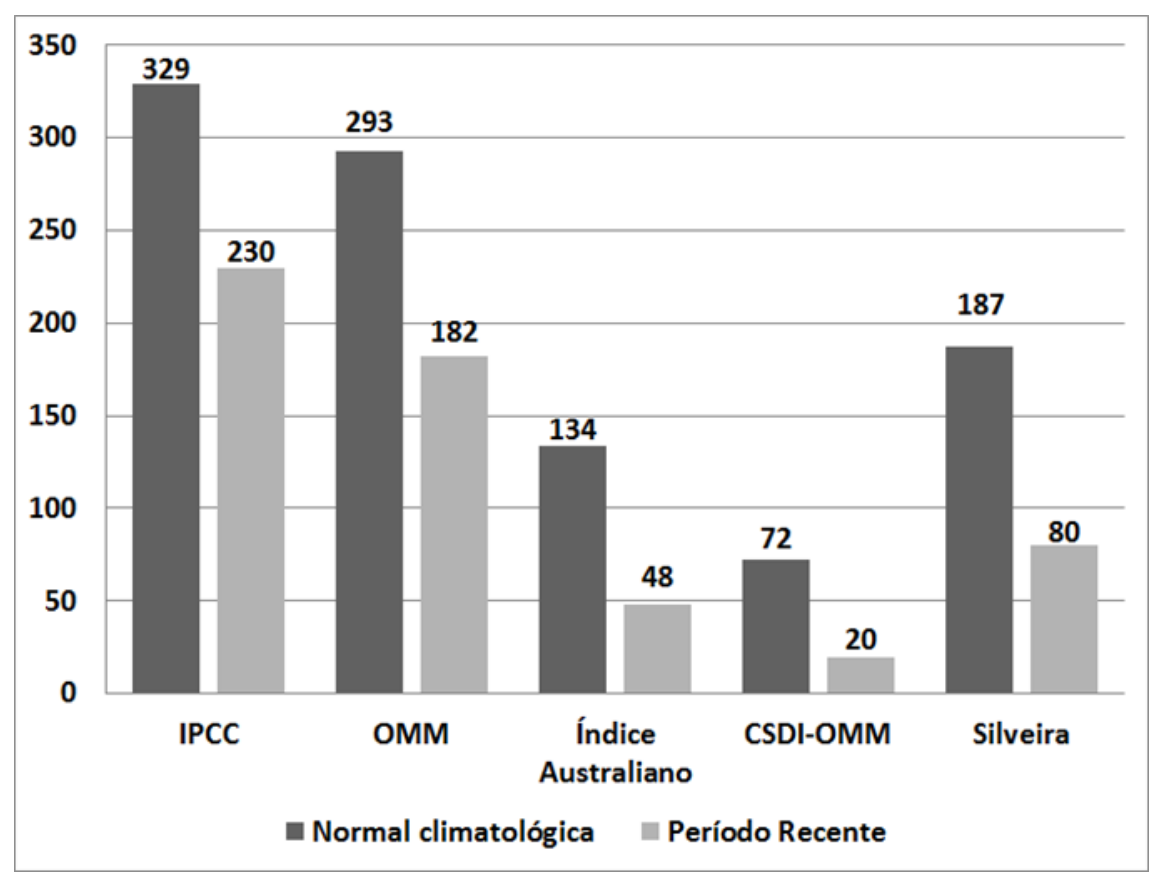

Figura 4: Comparação dos totais de ondas de frio entre os diferentes índices climáticos e entre os períodos da normal climatológica (1961 - 1990) e recente (1991 - 2014). Fonte: Estação Meteorológica da ESALQ/USP. Organização: Fante e Armond (2016).

Apesar de o período recente ter um número menor de anos (23 anos) quando comparado à normal climatológica (30 anos), é inegável a discrepância do total de ocorrências de eventos extremos frio nos dois períodos. Foram constatadas as diminuições de 111 ondas de frio durante o período recente, de acordo com o índice da OMM; menos 107 ondas de frio de acordo com a técnica de Silveira (2013); menos 99 ondas para a técnica de Silveira (2013); menos 86 ondas constatadas com o índice australiano e, por fim, menos 52 ondas de frio verificadas pelo índice de CSDI-OMM.

Em relação aos dados de internações por doenças respiratórias, obtidos no banco de dados do DataSUS observa-se a ocorrência de mais de 10 mil internações entre os anos de 1998 e 2014 (Figura 5).

Uma análise mais minuciosa da faixa etária demonstra que a população de 0 a 9 anos e acima de 80 anos representam mais da metade de todas as internações. A maior vulnerabilidade dessa faixa etária a doenças do aparelho respiratório também foi encontrada em Souza (2008), Aleixo (2012) e Araújo (2014). 
Para Araújo (2014) inclusive, os idosos e crianças são os mais vulneráveis devido a fragilidade do seu estado de saúde, falta de autonomia física e psíquica e, no caso dos idosos, patologias crônicas. As condições econômicas de sobrevivência dessas faixas etárias seriam fundamentais para intensificação ou amenização dos níveis de vulnerabilidade a doenças do aparelho respiratório.

No que se refere à distribuição mensal das internações, as maiores médias encontram-se nos meses da estação menos chuvosa (de abril a outubro), com destaque para o mês de julho que apresentou média superior a 200 internações.

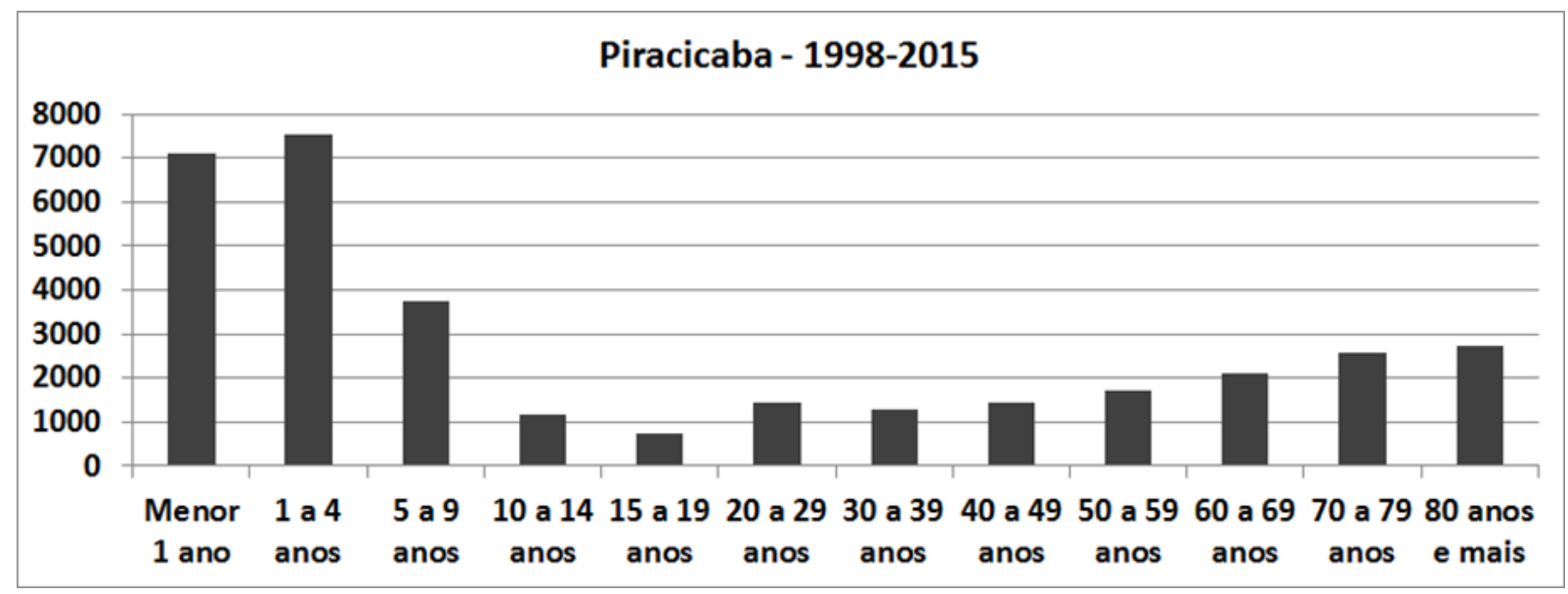

Figura 5: Total de internações por doenças respiratórias, segundo faixa etária. Fonte: DataSUS, 2015. Organização: Fante e Armond (2016).

A sazonalidade climática, nesse contexto, se relaciona com as médias de internações, como também foi encontrado em Souza (2008), Aleixo (2012) e Araújo (2014). Segundo Souza (2008), as condições de estabilidade atmosférica (que se dão, sobretudo, nos meses de inverno) são desfavoráveis a dispersão de poluentes. Condição inversa ocorre quando do domínio de sistemas instáveis, durante os quais as chuvas 'lavam' a atmosfera, reduzindo os níveis de contaminantes e a dinâmica de ventos atua dispersando os poluentes (Figura 6).

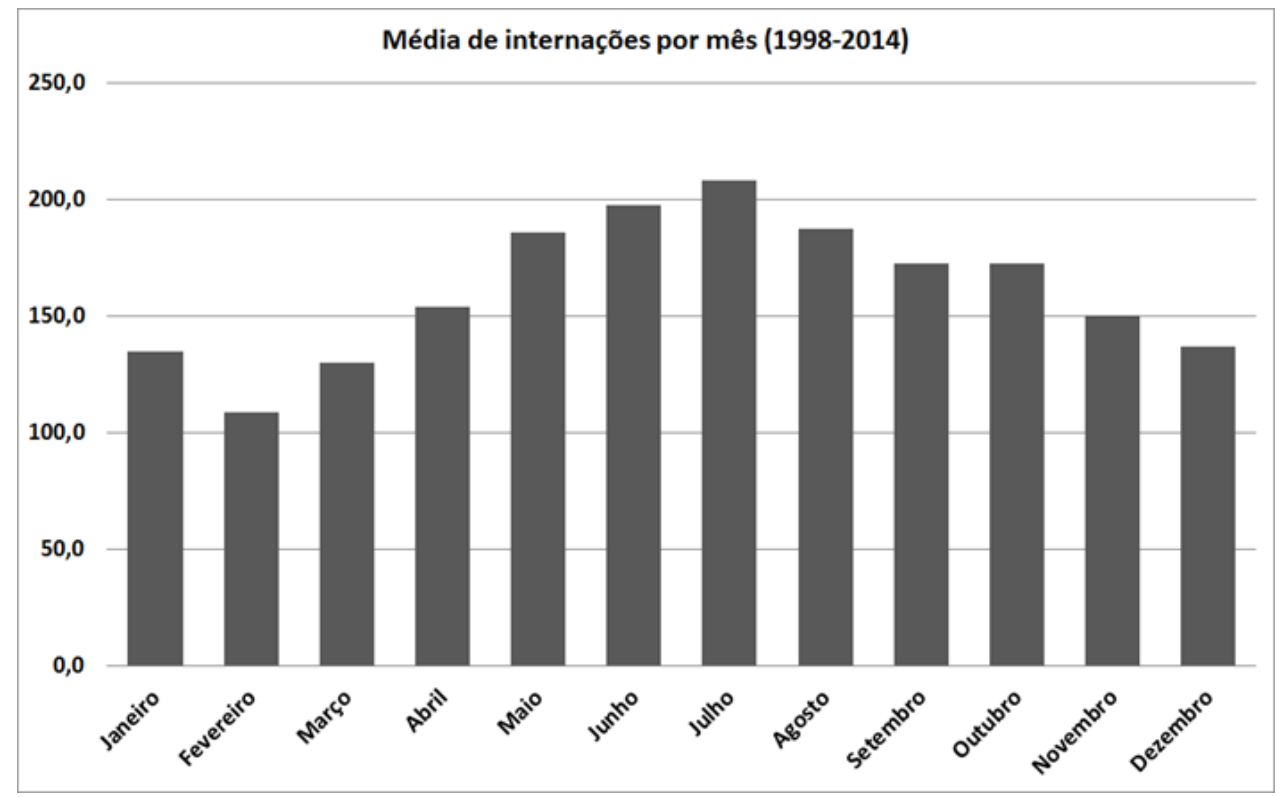

Figura 6: Média mensal de internações por doenças respiratórias (1998-2014). Fonte: DataSUS. Organização: Fante e Armond (2016). 
Observa-se nos gráficos supracitados que a maior incidência dos eventos de ondas de frio e do número de internações ocorre durante os meses representativos de inverno, na cidade de Piracicaba.

No entanto, quando se analisa os mesmos gráficos na escala anual, verifica-se que nem sempre um ano considerado frio, com elevado número de ondas de frio, é o suficiente para desencadear maior número de internações com gêneses em enfermidades respiratórias.

Como pode ser observada na Figura 7, a sobreposição e comparação do resultado da ocorrência das ondas de frio e dos totais anuais de internação não apresentou correlação significativa em nenhum dos índices climáticos utilizados na pesquisa.

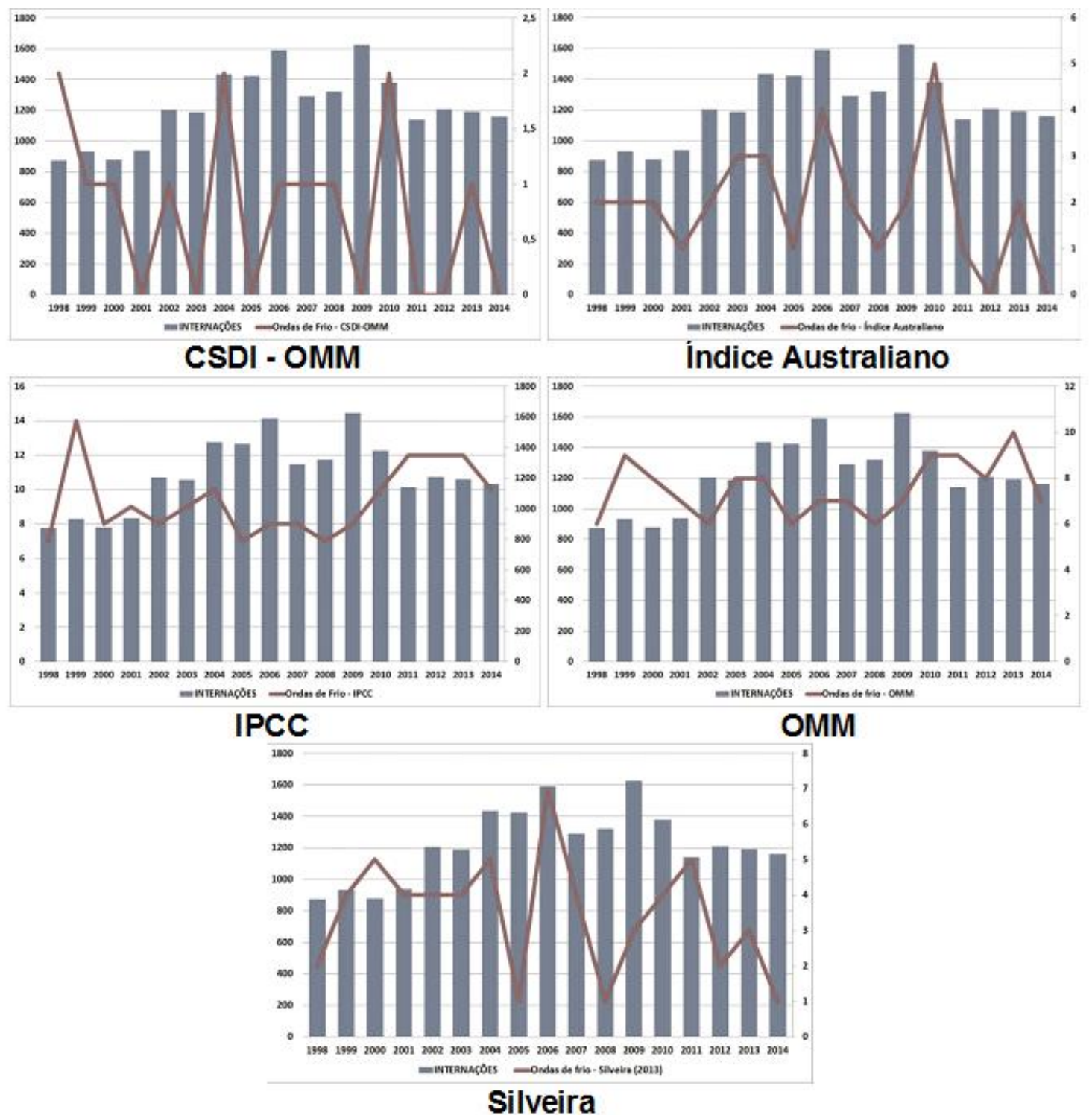

Figura 7: Total de internações por doenças respiratórias (barras) e o número de ondas de frio (linhas), segundo índices climáticos Fonte: DataSUS e Estação Meteorológica da ESALQ/USP. Organização: Fante e Armond (2016).

Os anos com maior número de internações, 2009 e 2006, por exemplo, não foram, para nenhuma das cinco técnicas utilizadas, anos com expressiva ocorrência de ondas de frio. Tal fato remete a hipótese de que alguns detalhes devem ser mais bem analisados para que os resultados sejam mais explícitos.

De todo modo, é inegável o papel das temperaturas na deflagração de enfermidades de cunho respiratório, no entanto, não apenas esse elemento atmosférico é o responsável para a ocorrência de maior ou menor número de internações. 
No caso das ondas de frio, as técnicas evidenciam o valor excepcional a partir de limiares de dias de permanência. No entanto, a ontologia da doença vai além desses pressupostos. Nem todo evento extremo (flutuação estatística acima dos padrões habituais) efetivamente deflagra situações como internações.

Há de se considerar que a repercussão negativa da doença está intimamente ligada à vulnerabilidade do indivíduo, tanto no quesito biológico, fragilidade física e etária, quanto na sua condição econômica e social de se precaver e proteger das situações climáticas anômalas

Além disso, é importante destacar que alguns fatores bioclimáticos também tem um papel fundamental no desencadeamento positivo ou negativo da doença como a exposição à umidade, circulação dos ventos e a própria quantidade e qualidade dos materiais particulados presentes na atmosfera.

Neste sentido, apenas um estudo microclimático e dentro do interior das residências pode ser capaz de inferir os principais motivos dessas deflagrações. É nesta escala que a vulnerabilidade pode ser mais bem compreendida explicitando as diferentes condições de exposição e de fragilidade dos diferentes grupos sociais aos eventos extremos.

Torna-se quase inócuo tratar somente dos riscos aos quais as populações urbanas estão submetidas aos eventos extremos apenas na perspectiva quantitativa, pois eles se explicitam diferente sobre grupos extremamente heterogêneos no interior da sociedade. Desta forma, evento extremo e vulnerabilidade revelase como um híbrido, intrínseco, dialético e indissociável.

Outro ponto principal que merece ser ressaltado nesta análise é a natureza dos dados. O DataSUS disponibiliza apenas informações mensais das internações, enquanto as ondas de frio ocorrem na escala diária/semanal. Há de se considerar que, no caso das enfermidades respiratórias, não existe uma relação de causa-efeito, mas é evidente toda uma gradação de agravamento e piora nos sintomas até que o paciente seja internado. É inequívoco o delay entre o momento precursor e a morbidade respiratória.

\section{CONCLUSÕES}

Para estabelecer a relação entre as ondas de frio e a vulnerabilidade da população, foram analisados dados quantitativos com ênfase em aspectos relativos à temperatura e internações por enfermidades respiratórias.

No âmbito dos dados climáticos utilizados para delimitar as ondas de frio, elas foram comparadas de acordo com cada índice e entre dois períodos: normal climatológica $(1961$ - 1990) e período recente (1991 2014). As curvas de ocorrência apresentaram convergência entre as técnicas. O período recente (23 anos) apresentou menor total de ondas de frio do que o período da normal climatológica (30 anos).

Foi encontrada considerável variação dos totais de ondas de frio de acordo com cada uma das técnicas empregadas, entre 72 e 329 ondas para o mesmo período. Essa diferença diz respeito à natureza das variáveis empregadas, que dão peso maior ou à duração da onda, ou à intensidade, ou à frequência. A partir dos dados de internação por enfermidades respiratórias, depreendeu-se que a população mais vulnerável a esse tipo de doença encontra-se na faixa etária entre 0 a 9 anos e acima de 80 anos.

O maior número de internações se deu nos meses da estação menos chuvosa (fim do outono até o início da primavera), com destaque para o mês de julho. A sazonalidade atrelada à dinâmica atmosférica apresenta, portanto, influência nas internações. Entretanto, quando foram comparados os dados de ocorrência das ondas de frio (totais mensais) e os totais de internações (também em escala mensal), os resultados não apresentaram convergência e, por vezes, foram até mesmo discrepantes. Esse resultado fez com que algumas reflexões sobre os procedimentos metodológicos e o banco de dados fossem suscitadas.

As formas pelas quais o banco de dados utilizado estava estruturado contribuem para a ausência de correlação entre as variáveis. Considera-se a possibilidade de ocorrência de dois problemas escalares; um, de ordem temporal, e outro de ordem espacial.

No aspecto temporal os dados do DataSUS em escala mensal impossibilitaram a comparação em escala diária de ocorrência das ondas de frio. Os dados na escala mensal podem mascarar as possibilidades de investigação das diferentes formas pelas quais se dão as relações entre as condições do tempo atmosférico 
e deflagração da enfermidade. Neste caso há de se considerar, por exemplo, delays entre a ocorrência da onda de frio e o desencadeamento da internação por doença respiratória. Estudos como os de Bell et al. (2004), Carder et al. (2005), Analitis et al. (2008), Anderson e Bell (2009) e Gasparrini (2016), dispondo de uma série de dados diários de internações puderam explorar as diferentes interações temporais com os dados meteorológicos. Em suma, a relação entre o tempo (meteorológico) e o corpo humano/aparelho respiratório não se dá em curta escala temporal, e tampouco ocorre de forma linear, como causa-efeito.

Em escala espacial, os dados de temperatura da estação meteorológica da ESALQ/USP não representam, necessariamente, a temperatura dentro das residências da população de Piracicaba. A análise dos elementos do clima em escala microclimática (conforto térmico) pode se apresentar mais eficaz e fidedigna quando relacionada com morbidades e enfermidades. Variáveis como os tipos de materiais construtivos, circulação de ar no interior das residências, as medidas adaptativas ao frio, densidade de habitantes por residência, dentre outras, são fundamentais para uma análise complexa dessa relação. A vulnerabilidade da população a ondas de frio demanda a consideração de fatores que vão para além do clima. Em síntese, considera-se que a relação entre ondas de frio e morbidade respiratória da população deve contabilizar aspectos de várias naturezas para a definição da vulnerabilidade.

\section{REFERÊNCIAS}

ADGER. N. Vulnerability. Global environmental change. v. 16, p. 268-281, 15 February 2006.

ALEIXO, N. C. Pelas lentes da climatologia e da saúde pública: doenças hídricas e respiratórias na cidade de Ribeirão Preto/SP. 2012. 353 f. Tese (doutorado). Faculdade de Ciências e Tecnologia. Universidade Estadual Paulista, Presidente Prudente/SP.

ANALITIS, A.; KATSOUYANNI, K.; BIGGERI, A. et al. Effects of cold wather on mortality: results from 15 European cities within the PHEWE Project. American Journal of Epidemiology, v. 168, p. 1397, 2008.

ANDERSON, B.G.; BELL, M.L. Weather-related mortality: how heat, cold, and heat waves affect mortality in United States. Epidemiology, v. 20, p. 205-213, 2009.

ARAÚJO, R.R. Clima e vulnerabilidade socioespacial: uma avaliação dos fatores de risco na saúde da população urbana do município de São Luís (MA). 2014, 289 f. Tese (doutorado) - Faculdade de Ciências e Tecnologia. Universidade Estadual Paulista, Presidente Prudente/SP. [s.n],

ARMOND, N. B. Entre eventos e episódios: as excepcionalidades das chuvas e os alagamentos no espaço urbano do Rio de Janeiro. 2014, 239f. Dissertação (mestrado). Faculdade de Ciências e Tecnologia. Universidade Estadual Paulista, Presidente Prudente/SP.

BELL, M.L.; McDERMOTT, A.; ZEGER, S.L.; DOMINICI, F. Ozone and short-term mortality in 95 US urban communities, 1987-2000. Journal of American Meteorological Society, v. 292, p. 2372-2378, 2004.

BRAGA, A. L. F.; PEREIRA, L. A. A.; PROCÓPIO, M.; ANDRÉ, P. A. de ; SALDIVA, P. H. do N. Associação entre poluição atmosférica e doenças respiratórias e cardiovasculares na cidade de Itabira, Minas Gerais, Brasil. Cad. Saúde Pública, Rio de Janeiro, 23 Sup 4 :p. 570-578, 2007. Disponível em <http://www.scielo.br/pdf/csp/v23s4/09.pdf> Acesso em 07 ago. 2015.

CARDER, M.; McNAMEE, R.; BEVERLAND, I. et al. The lagged effect of cold temperature and wind chill on cardiorespiratory mortality in Scotland. Occup. Environ. Med., v. 62, p. 702-710, 2005.

CIDADE, L.F.C. Urbanização, ambiente, risco e vulnerabilidade: em busca de uma construção interdisciplinar. Cadernos Metrópole, São Paulo, v. 15, n. 29, p. 171-191, jan/jun 2013.

COLLINS, J. M.; CHAVES, R. R.; MARQUES, V. da S. Temperature Variability over South America. Journal of climate. v. 22, p. $5854-5869,15$ de November, 2009.

CONFALONIERI, U.E.C. Variabilidade climática, vulnerabilidade social e saúde no Brasil. Terra Livre, São Paulo. vol. 19, n. 20, p. 193-204, jan/jul 2003.

CUTTER, S. Vulnerability to environmental hazards. Progress in Human Geography, Nova York, v. 20, n 4, p. 529-539, December 1996. 
The vulnerability of science and the science of vulnerability. Annals of the Association of American Geographers, Oxford. v. 93, n¹, p. 1-12, March 2003.

DADOS CLIMATOLÓGICOS. Escola Superior de Agricultura Luiz de Queiroz da Universidade de São Paulo (ESALQ/USP). Disponível em <http://www.leb.esalq.usp.br/anos.html> Acessos em 01 jun. 2015.

DOW, K. Exploring differences in our common future(s): the meaning of vulnerability to global environmental change. Geoforum, vol. 23, n. 3, p. 417-436, 1992.

DUFEK, A. S.; AMBRIZZI, T. Variabilidade climática da temperatura no Estado de São Paulo. Revista de Iniciação Científica. (USP), São Paulo/SP. v.7, p. 23-29. 2005.

FANTE, K. P. Variabilidade da temperatura em áreas urbanas não metropolitanas do Estado de São Paulo Brasil no período de 1961 a 2011. 2014, 260 f. Dissertação de Mestrado em Geografia. Faculdade de Ciências e Tecnologia. Universidade Estadual Paulista, Presidente Prudente/SP.

FERRAZ, F. F., FERRAZ, E. S., BALLESTER, M. V. R., MORAES, J., VICTORIA, R. L; MARTINELLI, L. A. Previsão de áreas inundadas na cidade de Piracicaba (SP) através de sistema de informações geográficas (SIG). Revista Brasileira de Recursos hídricos. Porto Alegre/RS. vol 3, n. 3, p. 17-27, Jul/Set $1998 . \quad$ Disponível em: <http://www.abrh.org.br/SGCv3/UserFiles/Sumarios/87bdd48afe86131189e2e16ecc85b7cd_3763d1349fc2 d35783bd639dfac2c517.pdf > Acessado em: 28/08/2014.

FREITAS, C. U. de et al. Poluição do ar em cidades brasileiras: selecionando indicadores de impacto na saúde para fins de vigilância. Epidemiol. Serv. Saúde, Brasília, v. 22, n. 3, set. 2013. Disponível em < http://scielo.iec.pa.gov.br/scielo.php?script=sci_arttext\&pid=S1679-

49742013000300009\&lng=pt\&nrm=iso>. Acessos em 07 ago. 2015. http://dx.doi.org/10.5123/S167949742013000300009 .

GASPARRINI, A. Modelling lagged associations in environmental time series data: a simulation study. Epidemiology, v. 27, n. 6, p. 835-842, 2016.

LUERS, A. The surface of vulnerability: an analytical framework for examining environmental change. Global environmental change, v. 15, p. 214-223, October 2005.

MINUZZI, R. B.; CARAMORI, P. H.; BORROZINO, E. Tendências na variabilidade climática sazonal e anual das temperaturas máxima e mínima do ar no Estado do Paraná. Bragantina. Campinas/SP. n.2, v. 70, p. $471-479,2011$.

MONTEIRO, A.; CARVALHO, V. Uma abordagem metodológica para avaliação de eventos climáticos extremos. In: AMORIM, M. C. T. de.; SANT'ANNA NETO, J. L.; MONTEIRO, A. (org) Climatologia urbana e regional: questões teóricas e estudos de caso. $1^{\mathrm{a}}$ edição. São Paulo: Outras expressões- Geografia em Movimento, 2013. p. 117 - 142.

MONTEIRO, C.A.F. Clima e excepcionalismo. Conjecturas sobre o desempenho da atmosfera como fenômeno geográfico. Florianópolis: Editora da UFSC, 1991/ 241 p.

MURARA, P. G. S. Clima e saúde. Variações atmosféricas e enfermidades circulatórias. 2009, 80 f. Trabalho de conclusão (bacharelado - Geografia) - Faculdade de Ciências e Tecnologia. Universidade Estadual Paulista, Presidente Prudente/SP.

Variabilidade Climática e Doenças Circulatórias e Respiratórias em Florianópolis (SC): uma contribuição à Climatologia Médica. 2012. 94 f. Dissertação (mestrado) - Centro de Filosofia e Ciências Humanas. Universidade Federal de Santa Catarina. Programa de Pós-Graduação em Geografia. Florianópolis/SC. Disponível em <https://repositorio.ufsc.br/bitstream/handle/123456789/99358/307856.pdf?sequence=1> Acessos em 07 ago. 2015.

NIMER, E. Climatologia do Brasil. Rio de Janeiro: IBGE, 1989.

NOGUEIRA, J. B. Poluição Atmosférica e Doenças Cardiovasculares. Revista Portuguesa de Cardiologia. Lisboa. Vol. 28, p. 715-733. Junho 2009. Disponível em <http://www.spc.pt/DL/RPC/artigos/1080.pdf> Acessos em 07 ago. 2015. 
SANT'ANNA NETO, J.L. Por uma Geografia do Clima - antecedentes históricos, paradigmas contemporâneos e uma nova razão para um novo conhecimento. Terra Livre, São Paulo/SP. v. 17, p. 49$62,2^{\circ}$ semestre, 2001.

. O decálogo da climatologia do Sudeste brasileiro. Revista Brasileira de Climatologia, Presidente Prudente/SP. v.1, n.1, pp. 43-60, Dezembro 2005.

Da climatologia geográfica à geografia do clima: gênese, paradigmas e aplicações do clima como fenômeno geográfico. Revista da ANPEGE, v. 4, p. 1-18, 2008.

O clima urbano como construção social: da vulnerabilidade polissêmica das cidades enfermas ao sofisma utópico das cidades saudáveis. Revista Brasileira de Climatologia, Curitiba/PR. ano 7, vol. 8, p. 45-60, Jan/Jun 2011.

SATTERTHWAITE, D.; SALEEMUL, H.; REID, H.; PELling, M.; LANKAO, P.R. Adapting to climate change in urban areas: the possibilities and constraints in Low- and Middle-income nations. In: BICKNELL, J.; DODMAN, D.; SATTERTHWAITE, D. Adapting cities to climate change: understanding and addressing the development challenges. London: Earthscan, 2009.

SETTE, D. M.; RIBEIRO, H. Interações entre o clima, o tempo e a saúde humana. INTERFACEHS. Revista de saúde, meio ambiente e sustentabilidade. Local. $\mathrm{N}^{\circ} 2$, Volume 6, número 2, p. 37 - 51. Agosto 2011. Disponível em http://www3.sp.senac.br/hotsites/blogs/InterfacEHS/wpContent/uploads/2013/08/3_ARTIGO_vol6n2.pdf [ISSN 1980-0894] Acessos em 07 ago. 2015.

SILVA, E. N..; RIBEIRO, H.; SANTANA, P. Clima e saúde em contextos urbanos: uma revisão da literatura. Biblio 3W. Revista Bibliográfica de Geografía y Ciencias Sociales. Barcelona: Universidad de Barcelona, Vol. XIX, no 1092. 30 de octubre de 2014, Disponível em <http://www.ub.edu/geocrit/b3w1092.htm> [ISSN 1138-9796] Acessos em 07 ago. 2015.

SILVEIRA, R. D. Risco climático, vulnerabilidade socioespacial e eventos climáticos extremos relacionados ao calor e ao frio no Estado do Rio Grande do Sul - Brasil. 2013. 379 f. Tese de Doutorado. Faculdade de Ciências e Tecnologia, Universidade Estadual Paulista, Presidente Prudente/SP.

SMITH, K., PETLEY, D. Hazard in the environment. SMITH, K., PETLEY, D. In: Environmental Hazards. Assessing risk and reducing disaster. Fourth edition. New York: Routledge, 2007. p. 3-21, 2007.

SOUZA, C. G. A influência do ritmo climático na morbidade respiratória em ambientes urbanos. 2008, 200 f. Dissertação (mestrado). Faculdade de Ciências e Tecnologia, Universidade Estadual Paulista, Presidente Prudente.

SOUZA, C. G.; SANT’ANNA NETO, J. L. Ritmo Climático E Doenças Respiratórias: Interações E Paradóxos. Revista Brasileira de Climatologia. Presidente Prudente/SP. Volume 3 e 4. p. 65 - 82. Agosto -2008 .

VEYRET, Yvette. Os riscos. O homem como agressor e vítima do meio ambiente. $1^{\circ}$ Edição, São Paulo: Contexto, 2007. 319 p. 